\title{
Uniform oscillations of the local time of iterated Brownian motion
}

\author{
NATHALIE EISENBAUM ${ }^{1}$ and ZHAN SHI ${ }^{2}$ \\ ${ }^{1}$ Laboratoire de Probabilités, Université Paris VI, 4 place Jussieu, F-75252 Paris Cédex 05, \\ France.e-mail:nae@ccr.jussieu.fr \\ ${ }^{2}$ LSTA, Université Paris VI, 4 place Jussieu, F-75252 Paris Cédex 05, France. \\ e-mail:shi@ccr.jussieu.fr
}

Burdzy and Khoshnevisan in 1995, Csáki et al. in 1996 and Xiao in 1997 have given some interesting information about the modulus of continuity of the local time of iterated Brownian motion. The aim of this paper is to provide the exact rate functions, respectively for the modulus of continuity and for the modulus of non-differentiability. Our approach strongly relies on Ray-Knight theorems for Brownian local times and on fine properties of Bessel processes.

Keywords: iterated Brownian motion; local time; modulus of continuity; modulus of nondifferentiability

\section{Introduction}

Consider the real-valued process

$$
Z(t) \stackrel{\text { def }}{=}\left\{\begin{array}{ll}
X_{+}(Y(t)), & \text { if } Y(t) \geqslant 0, \\
X_{-}(Y(t)), & \text { if } Y(t)<0,
\end{array} \quad t \geqslant 0,\right.
$$

where $\left\{X_{+}(u) ; u \geqslant 0\right\},\left\{X_{-}(u) ; u \geqslant 0\right\}$ and $\{Y(t) ; t \geqslant 0\}$ are three independent onedimensional Brownian motions, starting from 0 . The study of $Z$, the so-called "iterated Brownian motion" process (terminology coined by Burdzy (1993)), has motivated several groups of mathematicians. We refer to $\mathrm{Hu}$ (1996, Chapter III) for a detailed survey. Let us also mention more recent work by Bertoin and Shi (1996), Csáki et al. (1996; 1997a,b), Csáki and Földes (1997), Khoshnevisan and Lewis (1997), Shi and Yor (1997) and Xiao (1997).

The present paper focuses on the local time of $Z$. It was proved independently by Burdzy and Khoshnevisan (1995) and Csáki et al. (1996) that there exists a jointly continuous version of $\left\{L_{t}^{x}(Z) ; t \geqslant 0, x \in \mathbb{R}\right\}$, the local time process of $Z$. Furthermore, it can be represented as

$$
L_{t}^{x}(Z)=\int_{0}^{\infty} L_{t}^{u}(Y) \mathrm{d}_{u} L_{u}^{x}\left(X_{+}\right)+\int_{0}^{\infty} L_{t}^{-u}(Y) \mathrm{d}_{u} L_{u}^{x}\left(X_{-}\right),
$$

where $L(\xi)$ denotes the local time of $\xi$, for any stochastic process $\xi$ indexed by $\mathbb{R}_{+}$. 
It is certainly interesting to study the (uniform) modulus of continuity of $t \mapsto L_{t}^{x}(Z)$, in the sense of Lévy (1939). Some interesting estimates were presented in the papers by Burdzy and Khoshnevisan (1995) and Csáki et al. (1996), which have been since improved by Xiao (1997). Let

$$
\omega(h) \stackrel{\text { def }}{=} \sup _{0 \leqslant t \leqslant 1-h} \sup _{x \in \mathbb{R}}\left\{L_{t+h}^{x}(Z)-L_{t}^{x}(Z)\right\}, \quad 0<h<1 .
$$

It was shown by Xiao (1997) that

$$
\limsup _{h \rightarrow 0} \frac{\omega(h)}{h^{3 / 4}|\log h|^{3 / 4}} \leqslant c_{1}, \quad \text { a.s., }
$$

for some (finite) universal constant $c_{1}>0$.

One may wonder if $h^{3 / 4}|\log h|^{3 / 4}$ is the correct rate function for $\omega(h)$. The answer is yes.

Theorem 1.1. Let $\omega(h)$ be as in (1.2). There exists a universal constant $c_{2}>0$ such that

$$
\liminf _{h \rightarrow 0} \frac{\omega(h)}{h^{3 / 4}|\log h|^{3 / 4}} \geqslant c_{2}, \quad \text { a.s. }
$$

The above provides an accurate picture of the modulus of continuity of $L(Z)$, up to multiplication by a constant. It is also natural to investigate the corresponding liminf behaviour of $L(Z)$, i.e. its "modulus of non-differentiability" in the sense of Csörgo" and Révész (1979). Define

$$
\eta(h) \stackrel{\text { def }}{=} \inf _{0 \leqslant t \leqslant 1-h} \sup _{x \in \mathbb{R}}\left\{L_{t+h}^{x}(Z)-L_{t}^{x}(Z)\right\}, \quad 0<h<1 .
$$

Our next result determines the exact order of magnitude of $\eta(h)$ when $h$ is small.

Theorem 1.2. Let $\eta(h)$ be as in (1.4); then

$$
c_{3} \leqslant \liminf _{h \rightarrow 0} \frac{|\log h|^{3 / 4}}{h^{3 / 4}} \eta(h) \leqslant \limsup _{h \rightarrow 0} \frac{|\log h|^{3 / 4}}{h^{3 / 4}} \eta(h) \leqslant c_{4}, \quad \text { a.s., }
$$

where $c_{3}>0$ and $c_{4}>0$ are absolute constants.

The rest of the paper is organized as follows. Section 2 is devoted to some preliminaries on Brownian local times and Bessel processes. Theorems 1.1 and 1.2 are proved in Sections 3 and 4 respectively.

Throughout the paper, unless stated otherwise, $c_{i}>0 \quad(1 \leqslant i \leqslant 30)$ denote absolute constants.

\section{Preliminaries}

Let $\{Y(t) ; t \geqslant 0\}$ as before denote standard one-dimensional Brownian motion, and let $\left\{R_{d}(t) ; t \geqslant 0\right\}$ be a $d$-dimensional $(d \geqslant 0)$ Bessel process. Unless stated otherwise, we shall 
assume that $R_{d}(0)=0$. For general properties of $R_{d}$, we refer to Revuz and Yor (1994, Chapter XI). We mention that, when $d$ is a positive integer, $R_{d}$ can be realized as the Euclidean modulus of $d$-dimensional Brownian motion.

Lemma 2.1. Fix $0<a<1$. There exists $c_{5}(a)>0$, depending only on a such that, for all $0<\epsilon \leqslant 1$,

$$
\mathbb{P}\left(\inf _{0 \leqslant x \leqslant \epsilon} L_{1}^{x}(Y)>\frac{a}{\epsilon}\right) \geqslant \exp \left(-\frac{c_{5}(a)}{\epsilon^{2}}\right)
$$

Proof. We only have to treat the situation when $\epsilon$ is in the neighbourhood of 0 . Consider the first hitting time of 1 by $Y$ :

$$
\sigma \stackrel{\text { def }}{=} \inf \{t>0: Y(t)=1\}
$$

Let $\Delta_{1}$ denote the probability term on the left-hand side of (2.1). By scaling, for any fixed constant $0<b<1$ (whose value is to be chosen later),

$$
\begin{aligned}
\Delta_{1} & =\mathbb{P}\left(\inf _{0 \leqslant x \leqslant b} L_{(b / \epsilon)^{2}}^{x}(Y)>\frac{a b}{\epsilon^{2}}\right) \\
& \geqslant \mathbb{P}\left(\inf _{0 \leqslant x \leqslant b} L_{\sigma}^{x}(Y)>\frac{a b}{\epsilon^{2}} ; \sigma<\frac{b^{2}}{\epsilon^{2}}\right) \\
& =\mathbb{P}\left(\inf _{0 \leqslant x \leqslant b} L_{\sigma}^{x}(Y)>\frac{a b}{\epsilon^{2}} ; \int_{0}^{1} L_{\sigma}^{1-x}(Y) \mathrm{d} x+\int_{0}^{\infty} L_{\sigma}^{-x}(Y) \mathrm{d} x<\frac{b^{2}}{\epsilon^{2}}\right) .
\end{aligned}
$$

According to the first Ray-Knight theorem (cf. for example, Rogers and Williams (1987, Theorem VI.52.1)), $\left\{L_{\sigma}^{1-x}(Y) ; x \geqslant 0\right\}$ is an inhomogeneous Markov process. More precisely, it is a two-dimensional squared Bessel process starting from 0 for $0 \leqslant x \leqslant 1$ and becomes a squared Bessel process of dimension 0 for $x \geqslant 1$. Hence,

$$
\Delta_{1} \geqslant \mathbb{P}\left(\inf _{1-b \leqslant t \leqslant 1} R_{2}^{2}(t)>\frac{a b}{\epsilon^{2}} ; \int_{0}^{1} R_{2}^{2}(t) \mathrm{d} t+\int_{0}^{\infty} U^{2}(t) \mathrm{d} t<\frac{b^{2}}{\epsilon^{2}}\right),
$$

where $U$ denotes a Bessel process of dimension 0 , starting from $U(0)=R_{2}(1)$. By scaling, if $\{V(t) ; t \geqslant 0\}$ stands for a Bessel process of dimension 0 with $V(0)=1$, independent of $R_{2}$, then

$$
\Delta_{1} \geqslant \mathbb{P}\left(\inf _{1-b \leqslant t \leqslant 1} R_{2}(t)>\frac{(a b)^{1 / 2}}{\epsilon} ; \int_{0}^{1} R_{2}^{2}(t) \mathrm{d} t+R_{2}^{4}(1) \int_{0}^{\infty} V^{2}(t) \mathrm{d} t<\frac{b^{2}}{\epsilon^{2}}\right) .
$$

Since $a<1$, we can choose $0<b<1, q>0$ and $r>v>u>1$ such that

$$
v^{2} a b(1-b)+r^{2} a b^{2}+q r^{4} a^{2} b^{2}<b^{2} .
$$


Consequently,

$$
\begin{aligned}
\Delta_{1} \geqslant \mathbb{P}\left(\int_{0}^{\infty} V^{2}(t) \mathrm{d} t<q \epsilon^{2}\right) \mathbb{P} & \left(R_{2}(t) \leqslant \frac{v(a b)^{1 / 2}}{\epsilon} \text { for all } 0 \leqslant t \leqslant 1-b ;\right. \\
\frac{(a b)^{1 / 2}}{\epsilon} & \left.\leqslant R_{2}(t) \leqslant \frac{r(a b)^{1 / 2}}{\epsilon} \text { for all } 1-b \leqslant t \leqslant 1\right) .
\end{aligned}
$$

Let us estimate $\mathbb{P}\left\{\int_{0}^{\infty} V^{2}(t) \mathrm{d} t<q \epsilon^{2}\right\}=\mathbb{P}_{1}\left\{\int_{0}^{\infty} R_{0}^{2}(t) \mathrm{d} t<q \epsilon^{2}\right\}$, where $\mathbb{P}_{x}$ denotes the probability under which $R_{d}$ starts from $x$ (thus $\left.\mathbb{P}_{0}=\mathbb{P}\right)$. Write $H_{0}(r) \stackrel{\text { def }}{=} \inf \{t \geqslant 0$ : $\left.R_{0}(t)=r\right\}$ for all $r \geqslant 0$. By the strong Markov property,

$$
\begin{aligned}
\mathbb{P}\left(\int_{0}^{\infty} V^{2}(t) \mathrm{d} t<q \epsilon^{2}\right) & \geqslant \mathbb{P}_{1}\left(H_{0}(0)<\frac{q \epsilon^{2}}{4} ; H_{0}(2)=\infty\right) \\
& \geqslant \mathbb{P}_{1}\left(H_{0}(0)<\frac{q \epsilon^{2}}{4}\right)-\mathbb{P}_{2}\left(H_{0}(0)<\frac{q \epsilon^{2}}{4}\right) .
\end{aligned}
$$

It is known that $H_{0}(0)$ under $\mathbb{P}_{r}$ is distributed as $\mathscr{L}_{4}(r) \stackrel{\text { def }}{=} \sup \left\{t>0: R_{4}(t)=r\right\}$ under $\mathbb{P}$ (cf. Revuz and Yor (1994, Exercise XI.1.23)). The law of the latter variable is calculated by Getoor (1979) (cf. also Khoshnevisan et al. (1994) and Yor (1992)):

$$
\frac{\partial}{\partial t} \mathbb{P}\left\{\mathscr{C}_{4}(r)<t\right\}=\frac{r^{2}}{2 t^{2}} \exp \left(-\frac{r^{2}}{2 t}\right) \mathbf{1}_{\{t>0\}} .
$$

Therefore, for all small $\epsilon$,

$$
\mathbb{P}\left(\int_{0}^{\infty} V^{2}(t) \mathrm{d} t<q \epsilon^{2}\right) \geqslant \frac{1}{2} \mathbb{P}_{1}\left(H_{0}(0)<\frac{q \epsilon^{2}}{4}\right)=\frac{1}{2} \exp \left(-\frac{2}{q \epsilon^{2}}\right) \geqslant \exp \left(-\frac{c_{6}(a)}{\epsilon^{2}}\right),
$$

where $c_{6}(a)>0$ denotes a constant depending only on $a$. Going back to (2.2) and using the Markov property,

$$
\begin{aligned}
\Delta_{1} \geqslant & \exp \left(-\frac{c_{6}(a)}{\epsilon^{2}}\right) \mathbb{P}\left(\sup _{0 \leqslant t \leqslant 1-b} R_{2}(t) \leqslant \frac{v(a b)^{1 / 2}}{\epsilon} ; R_{2}(1-b) \geqslant \frac{u(a b)^{1 / 2}}{\epsilon}\right) \\
& \times \inf _{u(a b)^{1 / 2} / \epsilon \leqslant x \leqslant v(a b)^{1 / 2} / \epsilon} \mathbb{P}_{x}\left(\frac{(a b)^{1 / 2}}{\epsilon} \leqslant R_{2}(t) \leqslant \frac{r(a b)^{1 / 2}}{\epsilon} \text { for all } 0 \leqslant t \leqslant b\right) .
\end{aligned}
$$

Recall that $R_{2}$ is the modulus of Brownian motion in $\mathbb{R}^{2}$. By Gaussian tail estimates, for each $s>0$,

$$
\begin{aligned}
\log \mathbb{P}\left(\sup _{0 \leqslant t \leqslant s} R_{2}(t) \geqslant \lambda\right) \sim-\frac{\lambda^{2}}{2 s}, \quad \lambda \rightarrow \infty, \\
\log \mathbb{P}\left\{R_{2}(s) \geqslant \lambda\right\} \sim-\frac{\lambda^{2}}{2 s}, \quad \lambda \rightarrow \infty,
\end{aligned}
$$


where the usual notation $a(x) \sim b(x)\left(x \rightarrow x_{0}\right)$ denotes $\lim _{x \rightarrow x_{0}}\{a(x) / b(x)\}=1$. Therefore, letting $\Delta_{2}$ denote the first probability term on the right-hand side of (2.3),

$$
\begin{aligned}
\Delta_{2} & \geqslant \mathbb{P}\left(R_{2}(1-b) \geqslant \frac{u(a b)^{1 / 2}}{\epsilon}\right)-\mathbb{P}\left(\sup _{0 \leqslant t \leqslant 1-b} R_{2}(t) \geqslant \frac{v(a b)^{1 / 2}}{\epsilon}\right) \\
& \geqslant \frac{1}{2} \mathbb{P}\left(R_{2}(1-b) \geqslant \frac{u(a b)^{1 / 2}}{\epsilon}\right) \\
& \geqslant \exp \left(-\frac{c_{7} u^{2} a b}{(1-b) \epsilon^{2}}\right) .
\end{aligned}
$$

On the other hand, by the scaling property, the infimum term on the right-hand side of (2.3) equals

$$
\inf _{u(a b)^{1 / 2} \leqslant x \leqslant v(a b)^{1 / 2}} \mathbb{P}_{x}\left\{(a b)^{1 / 2} \leqslant R_{2}(t) \leqslant r(a b)^{1 / 2} \text { for all } 0 \leqslant t \leqslant b \epsilon^{2}\right\}
$$

which is bounded below by a positive constant depending only on $a$. This, together with (2.3) and (2.4), yields Lemma 2.1.

Lemma 2.2. For $d>0$ and $x \geqslant 0$, let

$$
H_{d}(x) \stackrel{\text { def }}{=} \inf \left\{t \geqslant 0: R_{d}(t)=x\right\} .
$$

Whenever $r>0$,

$$
\int_{0}^{H_{d}(r)} R_{d}^{2}(t) \mathrm{d} t \stackrel{\text { law }}{=} H_{1+d / 2}\left(\frac{r^{2}}{2}\right),
$$

where $\stackrel{\text { law }}{=}$ denotes identity in distribution.

Proof. It is possible to directly prove Lemma 2.2 using Itô's calculus, but it turns out to be more convenient to apply the change of dimension theorem for Bessel processes given by Biane and Yor (1987) (cf. for example Revuz and Yor (1994, Proposition XI.1.11)) which asserts the existence of a coupling for $R_{d}$ and $R_{1+d / 2}$ such that

$$
2 R_{1+d / 2}^{1 / 2}(t)=R_{d}(A(t)), \quad t \geqslant 0,
$$

where $A(t) \stackrel{\text { def }}{=} \int_{0}^{t} \mathrm{~d} s / R_{1+d / 2}(s)$. Consequently,

$$
A^{-1}(t)=\frac{1}{4} \int_{0}^{t} R_{d}^{2}(s) \mathrm{d} s, \quad t \geqslant 0 .
$$

Accordingly, for each $x>0$, 


$$
\inf \left\{t>0: R_{1+d / 2}(t)=x\right\} \stackrel{\text { law }}{=} A^{-1}\left(H_{d}\left(2 x^{1 / 2}\right)\right)=\frac{1}{4} \int_{0}^{H_{d}\left(2 x^{1 / 2}\right)} R_{d}^{2}(s) \mathrm{d} s,
$$

which implies Lemma 2.2 by scaling and taking $x=r^{2} / 4$.

Lemma 2.3. There exist universal constants $c_{8}>0$ and $c_{9}>0$ such that, for any $0<\epsilon \leqslant 1$,

$$
\exp \left(-\frac{c_{8}}{\epsilon^{2}}\right) \leqslant \mathbb{P}\left\{\left(\sup _{x \in \mathbb{R}} L_{1}^{x}(Y)\right)^{2} \sup _{0 \leqslant t \leqslant 1}|Y(t)|<\epsilon\right\} \leqslant \exp \left(-\frac{c_{9}}{\epsilon^{2}}\right) .
$$

The proof of Lemma 2.3 is based on a Ray-Knight theorem due to Eisenbaum (1990). To be precise, let

$$
\alpha \stackrel{\text { def }}{=} \inf \left(t>0: \sup _{x \geqslant 0} L_{t}^{x}(Y)=1\right) .
$$

The following theorem characterizes the distribution of the process $\left\{L_{\alpha}^{x}(Y) ; x \in \mathbb{R}\right\}$. We write $\bar{Y}(\alpha) \stackrel{\text { def }}{=} \sup _{0 \leqslant t \leqslant \alpha} Y(t)$ for brevity.

Theorem 2.1 (Eisenbaum 1990). The random variable $L_{\alpha}^{0}(Y)$ is uniformly distributed in $(0,1)$. Moreover, given $L_{\alpha}^{0}(Y)=\lambda \in(0,1)$,

$$
\left\{L_{\alpha}^{-x}(Y) ; x \geqslant 0\right\},\left\{L_{\alpha}^{x}(Y) ; 0 \leqslant x \leqslant Y(\alpha)\right\}
$$

and

$$
\left\{L_{\alpha}^{\bar{Y}(\alpha)-x}(Y) ; 0 \leqslant x \leqslant \bar{Y}(\alpha)-Y(\alpha)\right\}
$$

are three independent processes, the first being a squared Bessel process of dimension 0, starting from $\lambda$, the second a two-dimensional squared Bessel process starting from $\lambda$ and killed when hitting 1 for the first time, and the third a four-dimensional squared Bessel process starting from 0 , killed when hitting 1 .

Proof of Lemma 2.3. Only the situation of small $\epsilon$ needs to be treated. The upper bound in (2.6) in easy. Indeed,

$$
\begin{aligned}
\sup _{x \in \mathbb{R}} L_{1}^{x}(Y) \sup _{0 \leqslant t \leqslant 1}|Y(t)| & \geqslant \frac{1}{2} \sup _{x \in \mathbb{R}} L_{1}^{x}(Y)\left(\sup _{0 \leqslant t \leqslant 1} Y(t)-\inf _{0 \leqslant t \leqslant 1} Y(t)\right) \\
& \geqslant \frac{1}{2} \int_{-\infty}^{\infty} L_{1}^{x}(Y) \mathrm{d} x \\
& =\frac{1}{2} .
\end{aligned}
$$

Now the upper bound in (2.6) follows from the second inequality in the following wellknown estimates due to Kesten (1965); cf. also Csáki and Földes (1986) for the best possible constants $c_{10}>0$ and $c_{11}>0$ :

$$
\exp \left(-\frac{c_{10}}{y^{2}}\right) \leqslant \mathbb{P}\left(\sup _{x \in \mathbb{R}} L_{1}^{x}(Y)<y\right) \leqslant \exp \left(-\frac{c_{11}}{y^{2}}\right), \quad 0<y \leqslant 1 .
$$


To verify the lower estimate in (2.6), let $\alpha$ be as in (2.7) and let $b>0$ be an absolute constant whose value is to be chosen later. Let $\Delta_{3}$ denote the probability term in (2.6). By scaling,

$$
\begin{aligned}
\Delta_{3} & =\mathbb{P}\left\{\left(\sup _{x \in \mathbb{R}} L_{b^{2} / \epsilon^{2}}^{x}(Y)\right)^{2} \sup _{0 \leqslant t \leqslant b^{2} / \epsilon^{2}}|Y(t)|<\frac{b^{3}}{\epsilon^{2}}\right\} \\
& \geqslant \mathbb{P}\left\{\left(\sup _{x \in \mathbb{R}} L_{\alpha}^{x}(Y)\right)^{2} \sup _{0 \leqslant t \leqslant \alpha}|Y(t)|<\frac{b^{3}}{\epsilon^{2}} ; \alpha \geqslant \frac{b^{2}}{\epsilon^{2}}\right\} \\
& \geqslant \mathbb{P}\left(\sup _{x \leqslant 0} L_{\alpha}^{x}(Y)<1 ; \sup _{0 \leqslant t \leqslant \alpha}|Y(t)|<\frac{b^{3}}{\epsilon^{2}} ; \alpha \geqslant \frac{b^{2}}{\epsilon^{2}} ; \frac{1}{3} \leqslant L_{\alpha}^{0}(Y) \leqslant \frac{1}{2}\right),
\end{aligned}
$$

where we have used the fact that $\sup _{x \geqslant 0} L_{\alpha}^{x}(Y)=1$. Let as before $\mathbb{P}_{x}$ denote the probability under which the $d$-dimensional Bessel process $R_{d}$ starts from $x$. Recall the definition of $H_{d}$ from (2.5). According to Theorem 2.1,

$$
\begin{aligned}
\Delta_{3} \geqslant & \int_{1 / 3}^{1 / 2} \mathbb{P}_{\lambda^{1 / 2}}\left(\sup _{t \geqslant 0} R_{0}(t)<1 ; H_{0}(0)<\frac{b^{3}}{\epsilon^{2}}\right) \mathbb{P}_{\lambda^{1 / 2}}\left(H_{2}(1)<\frac{b^{3}}{2 \epsilon^{2}}\right) \\
& \times \mathbb{P}\left(H_{4}(1)<\frac{b^{3}}{2 \epsilon^{2}} ; \int_{0}^{H_{4}(1)} R_{4}^{2}(t) \mathrm{d} t \geqslant \frac{b^{2}}{\epsilon^{2}}\right) \mathrm{d} \lambda .
\end{aligned}
$$

Obviously,

$$
\inf _{1 / 3 \leqslant \lambda \leqslant 1 / 2} \mathbb{P}_{\lambda^{1 / 2}}\left(\sup _{t \geqslant 0} R_{0}(t)<1 ; H_{0}(0)<\frac{b^{3}}{\epsilon^{2}}\right) \geqslant c_{12}, \quad \inf _{1 / 3 \leqslant \lambda \leqslant 1 / 2} \mathbb{P}_{\lambda^{1 / 2}}\left(H_{2}(1)<\frac{b^{3}}{2 \epsilon^{2}}\right) \geqslant c_{13} .
$$

As a consequence,

$$
\begin{aligned}
\Delta_{3} & \geqslant c_{14} \mathbb{P}\left(H_{4}(1)<\frac{b^{3}}{2 \epsilon^{2}} ; \int_{0}^{H_{4}(1)} R_{4}^{2}(t) \mathrm{d} t \geqslant \frac{b^{2}}{\epsilon^{2}}\right) \\
& \geqslant c_{14} \mathbb{P}\left(\int_{0}^{H_{4}(1)} R_{4}^{2}(t) \mathrm{d} t \geqslant \frac{b^{2}}{\epsilon^{2}}\right)-c_{14} \mathbb{P}\left(H_{4}(1) \geqslant \frac{b^{3}}{2 \epsilon^{2}}\right) \\
& =c_{14} \mathbb{P}\left(H_{3}\left(\frac{1}{2}\right) \geqslant \frac{b^{2}}{\epsilon^{2}}\right)-c_{14} \mathbb{P}\left(H_{4}(1) \geqslant \frac{b^{3}}{2 \epsilon^{2}}\right),
\end{aligned}
$$

by means of Lemma 2.2. Since $\mathbb{P}\left\{H_{d}(r)>x\right\}=\mathbb{P}\left\{\sup _{0 \leqslant t \leqslant x} R_{d}(t)<r\right\}$, we can use the following well-known estimate of Ciesielski and Taylor (1962): for fixed $r>0$ and $d>0$,

$$
\log \mathbb{P}\left\{H_{d}(r) \geqslant y\right\} \sim-\frac{j_{d / 2-1}^{2}}{2 r^{2}} y, \quad y \rightarrow \infty,
$$


where $j_{d / 2-1}$ is the smallest positive root of the Bessel function $J_{d / 2-1}$. Therefore, we can choose a sufficiently large constant $b$ such that

$$
\mathbb{P}\left(H_{3}\left(\frac{1}{2}\right) \geqslant \frac{b^{2}}{\epsilon^{2}}\right) \geqslant 2 \mathbb{P}\left(H_{4}(1) \geqslant \frac{b^{3}}{2 \epsilon^{2}}\right) \geqslant \exp \left(-\frac{c_{15}}{\epsilon^{2}}\right),
$$

which, jointly considered with (2.9), yields the lower estimate in Lemma 2.3.

\section{Proof of Theorem 1.1}

Let $Y$ be Brownian motion as before. Define a sequence of increasing stopping times $\left(\theta_{n}\right)_{n \geqslant 0}$ by recurrence: $\theta_{0} \stackrel{\text { def }}{=} 0$ and

$$
\theta_{n} \stackrel{\text { def }}{=} \inf \left\{t \geqslant 1+\theta_{n-1}: Y(t)=n\right\}, \quad n=1,2, \ldots
$$

Define the absolute constant

$$
c_{16} \stackrel{\text { def }}{=} \frac{1}{16 c_{5}^{1 / 4}},
$$

where $c_{5} \stackrel{\text { def }}{=} c_{5}(1 / 2)$ is introduced in (2.1) (taking $a=1 / 2$ in Lemma 2.1). In the rest of this section, we shall work only for large $T$. Define the random variable

$$
N \stackrel{\text { def }}{=} N(T)=\inf \left(n \geqslant 1: \theta_{n}>T-1\right) .
$$

Recall $\omega(h)$ from (1.2). By scaling,

$$
\begin{aligned}
\Delta_{4} & \stackrel{\text { def }}{=} \mathbb{P}\left\{\omega\left(\frac{1}{T}\right)<c_{16} T^{-3 / 4}(\log T)^{3 / 4}\right\} \\
& =\mathbb{P}\left(\sup _{0 \leqslant t \leqslant T-1} \sup _{y \in \mathbb{R}}\left\{L_{1+t}^{y}(Z)-L_{t}^{y}(Z)\right\}<c_{16}(\log T)^{3 / 4}\right) \\
& \leqslant \mathbb{P}(N \leqslant m)+\mathbb{P}\left(\max _{0 \leqslant n \leqslant m} \sup _{y \in \mathbb{R}}\left\{L_{1+\theta_{n}}^{y}(Z)-L_{\theta_{n}}^{y}(Z)\right\}<c_{16}(\log T)^{3 / 4}\right),
\end{aligned}
$$

where $m \stackrel{\text { def }}{=}\left[T^{1 / 4}\right]$, the integer part of $T^{1 / 4}$. Let us estimate the first probability term on the right-hand side. By definition,

$$
\mathbb{P}(N \leqslant m)=\mathbb{P}\left(\theta_{m}>T-1\right)=\mathbb{P}\left(\sum_{n=1}^{m}\left(\theta_{n}-\theta_{n-1}\right)>T-1\right) .
$$

Observe that $\left(\theta_{n}-\theta_{n-1}\right)_{n \geqslant 1}$ is a sequence of iid variables, distributed as $(1+\mathscr{N})^{2} / \tilde{N}^{2}$, where $\mathscr{N}$ and $\tilde{N}$ denote two independent Gaussian $\mathscr{N}(0,1)$ variables. Accordingly, for each $n$ and $\lambda>0$, 


$$
\begin{aligned}
\mathbb{P}\left(\theta_{n}-\theta_{n-1}>\lambda\right) & =\mathbb{P}\left\{(1+\mathscr{N})^{2}>\lambda \tilde{N}^{2}\right\} \\
& \leqslant \mathbb{P}\left(\mathscr{N}^{2}>\frac{1}{2} \lambda \tilde{N}^{2}-1\right) \\
& \leqslant \mathbb{P}\left(\mathscr{N}^{2}>\frac{1}{4} \lambda \tilde{N}^{2}\right)+\mathbb{P}\left\{\lambda \tilde{N}^{2}<4\right) \\
& \leqslant \mathbb{E}\left\{\exp \left(-\frac{1}{8} \lambda \tilde{N}^{2}\right)\right\}+\mathbb{P}\left(\lambda \tilde{N}^{2}<4\right) \\
& \leqslant \frac{c_{17}}{\lambda^{1 / 2}} .
\end{aligned}
$$

Therefore,

$$
\begin{aligned}
\mathbb{P}(N \leqslant m) & \leqslant \mathbb{P}\left(\max _{1 \leqslant n \leqslant m}\left(\theta_{n}-\theta_{n-1}\right)>\frac{T-1}{m}\right) \\
& =1-\prod_{n=1}^{m}\left\{1-\mathbb{P}\left(\theta_{n}-\theta_{n-1}>\frac{T-1}{m}\right)\right\} \\
& \leqslant 1-\left(1-\frac{c_{18}}{T^{3 / 8}}\right)^{m} \\
& \leqslant \frac{c_{19}}{T^{1 / 8}} .
\end{aligned}
$$

Going back to (3.2), we have

$$
\Delta_{4} \leqslant \frac{c_{19}}{T^{1 / 8}}+\mathbb{P}\left(\max _{0 \leqslant n \leqslant m} \sup _{y \in \mathbb{R}}\left\{L_{1+\theta_{n}}^{y}(Z)-L_{\theta_{n}}^{y}(Z)\right\}<c_{16}(\log T)^{3 / 4}\right) .
$$

It remains to estimate the probability term on the right-hand side. By (1.1), for any $b \stackrel{\text { def }}{=} b(T) \in(0,1)$,

$$
\begin{aligned}
\max _{0 \leqslant n \leqslant m} \sup _{y \in \mathbb{R}}\left\{L_{1+\theta_{n}}^{y}(Z)-L_{\theta_{n}}^{y}(Z)\right\} \\
\quad \geqslant \max _{0 \leqslant n \leqslant m} \sup _{y \in \mathbb{R}} \int_{0}^{\infty}\left\{L_{1+\theta_{n}}^{u}(Y)-L_{\theta_{n}}^{u}(Y)\right\} \mathrm{d}_{u} L_{u}^{y}\left(X_{+}\right) \\
\geqslant \max _{0 \leqslant n \leqslant m} \int_{n}^{n+b}\left\{L_{1+\theta_{n}}^{u}(Y)-L_{\theta_{n}}^{u}(Y)\right\} \mathrm{d}_{u} L_{u}^{X_{+}(n)}\left(X_{+}\right) \\
\geqslant \max _{0 \leqslant n \leqslant m} \inf _{0 \leqslant x \leqslant b}\left\{L_{1+\theta_{n}}^{x+n}(Y)-L_{\theta_{n}}^{x+n}(Y)\right\}\left\{L_{n+b}^{X_{+}(n)}\left(X_{+}\right)-L_{n}^{X_{+}(n)}\left(X_{+}\right)\right\} .
\end{aligned}
$$

By the strong Markov property, $\left\{L_{1+\theta_{n}}^{n+x}(Y)-L_{\theta_{n}}^{n+x}(Y) ; x \in \mathbb{R}\right\}_{n \geqslant 0}$ is an i.i.d. sequence of processes, distributed as $\left\{L_{1}^{x}(Y) ; x \in \mathbb{R}\right\}$. On the other hand, $\left\{L_{n+b}^{X_{+}(n)}\left(X_{+}\right)-L_{n}^{X_{+}(n)}\left(X_{+}\right)\right\}_{n \geqslant 0}$ are i.i.d. variables, distributed as $L_{b}^{0}\left(X_{+}\right)$. Consequently, 


$$
\begin{aligned}
\Delta_{4} & \leqslant \frac{c_{19}}{T^{1 / 8}}+\left[\mathbb{P}\left\{\left(\inf _{0 \leqslant x \leqslant b} L_{1}^{x}(Y)\right) L_{b}^{0}\left(X_{+}\right)<c_{16}(\log T)^{3 / 4}\right\}\right]^{m+1} \\
& \leqslant \frac{c_{19}}{T^{1 / 8}}+\left\{1-\mathbb{P}\left(\inf _{0 \leqslant x \leqslant b} L_{1}^{x}(Y)>\frac{1}{2 b}\right) \mathbb{P}\left\{L_{b}^{0}\left(X_{+}\right)>2 c_{16} b(\log T)^{3 / 4}\right\}\right\}^{m+1} .
\end{aligned}
$$

Now take $b \stackrel{\text { def }}{=}\left(16 c_{5} / \log T\right)^{1 / 2}$. Applying Lemma 2.1 gives

$$
\mathbb{P}\left(\inf _{0 \leqslant x \leqslant b} L_{1}^{x}(Y)>\frac{1}{2 b}\right) \geqslant \exp \left(-\frac{c_{5}}{b^{2}}\right)=\frac{1}{T^{1 / 16}},
$$

whereas by the usual Gaussian tail estimate and (3.1), for all large $T$,

$$
\begin{aligned}
\mathbb{P}\left\{L_{b}^{0}\left(X_{+}\right)>2 c_{16} b(\log T)^{3 / 4}\right\} & =\mathbb{P}\left\{|\mathcal{N}|>2 c_{16} b^{1 / 2}(\log T)^{3 / 4}\right\} \\
& \geqslant \exp \left\{-4 c_{16}^{2} b(\log T)^{3 / 2}\right\} \\
& =\frac{1}{T^{1 / 16}} .
\end{aligned}
$$

It follows that

$$
\Delta_{4} \leqslant \frac{c_{19}}{T^{1 / 8}}+\left(1-\frac{1}{T^{1 / 8}}\right)^{m+1} \leqslant \frac{c_{20}}{T^{1 / 8}} .
$$

Let $T_{k} \stackrel{\text { def }}{=} k^{9}$. By the Borel-Cantelli lemma,

$$
\liminf _{k \rightarrow \infty} \frac{\omega\left(1 / T_{k}\right)}{\varphi\left(1 / T_{k}\right)} \geqslant c_{16}, \quad \text { a.s., }
$$

where $\varphi(t) \stackrel{\text { def }}{=} t^{3 / 4}|\log t|^{3 / 4}$. Let $h \in\left[1 / T_{k+1}, 1 / T_{k}\right]$. By monotonicity, for large $k$,

$$
\frac{\omega\left(1 / T_{k+1}\right)}{\varphi\left(1 / T_{k}\right)} \leqslant \frac{\omega(h)}{\varphi(h)} \leqslant \frac{\omega\left(1 / T_{k}\right)}{\varphi\left(1 / T_{k+1}\right)} .
$$

Since $\varphi\left(1 / T_{k}\right) \sim \varphi\left(1 / T_{k+1}\right)$ as $k$ tends to infinity, this implies that

$$
\liminf _{h \rightarrow 0} \frac{\omega(h)}{\varphi(h)} \geqslant c_{16}, \quad \text { a.s., }
$$

as desired.

\section{Proof of Theorem 1.2}

Let us restate Theorem 1.2:

$$
\liminf _{h \rightarrow 0} \frac{|\log h|^{3 / 4}}{h^{3 / 4}} \eta(h) \geqslant c_{3}, \quad \text { a.s. }
$$




$$
\limsup _{h \rightarrow 0} \frac{|\log h|^{3 / 4}}{h^{3 / 4}} \eta(h) \leqslant c_{4}, \quad \text { a.s. }
$$

The proof is divided into two parts.

Proof of (4.1). Let $Z$ be the iterated Brownian motion in Section 1. Using Gaussian tail estimates, it is readily checked that, for $x \geqslant 1$,

$$
\mathbb{P}\left(\sup _{0 \leqslant s \leqslant 1} Z(s)>x\right) \leqslant \exp \left(-c_{21} x^{4 / 3}\right) .
$$

For more details, and for the best possible value of $c_{21}$, cf. Révész (1990, p. 126), Burdzy (1993) and Csáki et al. (1995).

By the occupation time formula,

$$
1=\int_{-\infty}^{\infty} L_{1}^{x}(Z) \mathrm{d} x \leqslant\left(\sup _{x \in \mathbb{R}} L_{1}^{x}(Z)\right)\left(\sup _{0 \leqslant s \leqslant 1} Z(s)-\inf _{0 \leqslant s \leqslant 1} Z(s)\right) .
$$

Therefore, by scaling, symmetry and (4.3), for any $h>0$ and $0<y \leqslant h^{3 / 4} / 2$,

$$
\begin{aligned}
\mathbb{P}\left(\sup _{x \in \mathbb{R}} L_{h}^{x}(Z)<y\right) & =\mathbb{P}\left(\sup _{x \in \mathbb{R}} L_{1}^{x}(Z)<\frac{y}{h^{3 / 4}}\right) \\
& \leqslant \mathbb{P}\left(\sup _{0 \leqslant s \leqslant 1} Z(s)-\inf _{0 \leqslant s \leqslant 1} Z(s)>\frac{h^{3 / 4}}{y}\right) \\
& \leqslant 2 \mathbb{P}\left(\sup _{0 \leqslant s \leqslant 1} Z(s)>\frac{h^{3 / 4}}{2 y}\right) \\
& \leqslant \exp \left(-c_{22} \frac{h}{y^{4 / 3}}\right) .
\end{aligned}
$$

Now let $h$ be very small, and define $t_{j} \stackrel{\text { def }}{=} j h|\log h|^{-3}$ for $0 \leqslant j \leqslant M(h) \stackrel{\text { def }}{=}\left[h^{-1}|\log h|^{3}\right]$. Introduce the absolute constant

$$
c_{23} \stackrel{\text { def }}{=}\left(\frac{c_{22}}{2}\right)^{3 / 4}
$$

Consider the probability

$$
\begin{aligned}
\Delta_{5} & \stackrel{\text { def }}{=} \mathbb{P}\left(\min _{0 \leqslant j \leqslant M(h)} \sup _{x \in \mathbb{R}}\left\{L_{t_{j}+h}^{x}(Z)-L_{t_{j}}^{x}(Z)\right\}<c_{23} \frac{h^{3 / 4}}{|\log h|^{3 / 4}}\right) \\
& \leqslant \sum_{j=0}^{M(h)} \mathbb{P}\left(\sup _{x \in \mathbb{R}}\left\{L_{t_{j}+h}^{x}(Z)-L_{t_{j}}^{x}(Z)\right\}<c_{23} \frac{h^{3 / 4}}{|\log h|^{3 / 4}}\right) .
\end{aligned}
$$

Since the iterated Brownian motion $Z$ has stationary (but not independent) increments, for 
each fixed $t \geqslant 0, \sup _{x \in \mathbb{R}}\left\{L_{t+h}^{x}(Z)-L_{t}^{x}(Z)\right\}$ has the same law as $\sup _{x \in \mathbb{R}} L_{h}^{x}(Z)$. By (4.4) and (4.5),

$$
\Delta_{5} \leqslant\{M(h)+1\} \exp \left\{-\frac{c_{22}}{c_{23}^{4 / 3}} \log \left(\frac{1}{h}\right)\right\} \leqslant c_{24} h|\log h|^{3} .
$$

Let $h_{k} \stackrel{\text { def }}{=} k^{-2}$. It follows from the Borel-Cantelli lemma that

$$
\liminf _{k \rightarrow \infty} \frac{\left|\log h_{k}\right|^{3 / 4}}{h_{k}^{3 / 4}} \min _{0 \leqslant j \leqslant M\left(h_{k}\right)} \sup _{x \in \mathbb{R}}\left\{L_{t_{j}+h_{k}}^{x}(Z)-L_{t_{j}}^{x}(Z)\right\} \geqslant c_{23}, \quad \text { a.s., }
$$

where we have written $t_{j} \stackrel{\text { def }}{=} j h_{k}\left|\log h_{k}\right|^{-3}$ by (slight) abuse of notation. On the other hand, applying (1.3) readily gives

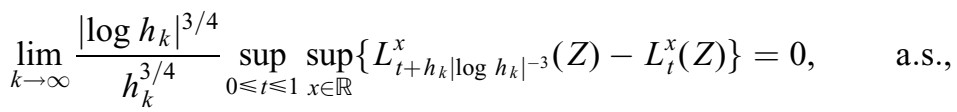

which, in view of (4.6), yields (recalling $\eta(h)$ from (1.4))

$$
\liminf _{k \rightarrow \infty} \frac{\left|\log h_{k}\right|^{3 / 4}}{h_{k}^{3 / 4}} \eta\left(h_{k}\right) \geqslant c_{23}, \quad \text { a.s. }
$$

This yields (4.1) in view of monotonicity of the function $h \mapsto|\log h|^{3 / 4} / h^{3 / 4}$.

Proof of (4.2). By (2.8) and Lemma 2.3, there exists a universal constant $c_{25}>0$ such that, for all $0<\lambda \leqslant 1$,

$$
\begin{gathered}
\mathbb{P}\left(\sup _{x \in \mathbb{R}} L_{1}^{x}\left(X_{+}\right)<\lambda\right) \geqslant \exp \left(-\frac{c_{25}}{\lambda^{2}}\right), \\
\mathbb{P}\left\{\left(\sup _{x \in \mathbb{R}} L_{1}^{x}(Y)\right)^{2}\left(\sup _{0 \leqslant t \leqslant 1} Y(t)-\inf _{0 \leqslant t \leqslant 1} Y(t)\right)<\lambda\right\} \geqslant \exp \left(-\frac{c_{25}}{\lambda^{2}}\right) .
\end{gathered}
$$

Pick the absolute constant

$$
c_{26} \stackrel{\text { def }}{=}\left(32 c_{25}\right)^{3 / 4}
$$

For notational simplification, we write the "two-sided local time" process

$$
L_{u}^{x}(X) \stackrel{\text { def }}{=} \begin{cases}L_{u}^{x}\left(X_{+}\right), & \text {if } u \geqslant 0 \\ -L_{-u}^{x}\left(X_{-}\right), & \text {otherwise }\end{cases}
$$

Then (1.1) can be simply written as

$$
L_{t}^{x}(Z)=\int_{-\infty}^{\infty} L_{t}^{u}(Y) \mathrm{d}_{u} L_{u}^{x}(X), \quad x \in \mathbb{R} .
$$

Fix a sufficiently large $T$ and define a sequence of stopping times $\left(\tau_{n}\right)_{n \geqslant 0}$ by $\tau_{0} \stackrel{\text { def }}{=} 0$ and 


$$
\tau_{n} \stackrel{\text { def }}{=} \tau_{n}(T)=\inf \left\{t \geqslant 1+\tau_{n-1}: Y(t)=n T^{1 / 16}\right\}, \quad n=1,2, \ldots
$$

Note the similarity between the $\tau_{n}$ and $\theta_{n}$ introduced in Section 3. Define

$$
\begin{aligned}
& m \stackrel{\text { def }}{=}\left[T^{1 / 8}\right] \\
& N \stackrel{\text { def }}{=} \inf \left(n \geqslant 1: \tau_{n}>T-1\right), \\
& E \stackrel{\text { def }}{=}(N \geqslant m+1) \cap \bigcap_{n=1}^{N}\left(\inf _{\tau_{n} \leqslant t \leqslant 1+\tau_{n}} Y(t) \geqslant \sup _{\tau_{n-1} \leqslant t \leqslant 1+\tau_{n-1}} Y(t)\right) .
\end{aligned}
$$

Consider the probability

$$
\begin{aligned}
\Delta_{6} & \stackrel{\text { def }}{=} \mathbb{P}\left\{\eta\left(\frac{1}{T}\right)>c_{26} T^{-3 / 4}(\log T)^{-3 / 4}\right\} \\
& =\mathbb{P}\left(\inf _{0 \leqslant t \leqslant T-1} \sup _{x \in \mathbb{R}}\left\{L_{t+1}^{x}(Z)-L_{t}^{x}(Z)\right\}>c_{26}(\log T)^{-3 / 4}\right) \\
& \leqslant \mathbb{P}\left(\min _{0 \leqslant n \leqslant N-1} \sup _{x \in \mathbb{R}}\left\{L_{1+\tau_{n}}^{x}(Z)-L_{\tau_{n}}^{x}(Z)\right\}>c_{26}(\log T)^{-3 / 4}\right) \\
& \leqslant \mathbb{P}\left(E^{c}\right)+\mathbb{P}\left(E ; \min _{1 \leqslant n \leqslant N-1} \sup _{x \in \mathbb{R}}\left\{L_{1+\tau_{n}}^{x}(Z)-L_{\tau_{n}}^{x}(Z)\right\}>c_{26}(\log T)^{-3 / 4}\right) \\
& \stackrel{\text { def }}{=} \mathbb{P}\left(E^{c}\right)+\Delta_{7},
\end{aligned}
$$

with obvious notation. For brevity, for any $0 \leqslant s \leqslant t$ and $-\infty<u \leqslant v<\infty$, write

$$
\begin{gathered}
L_{Y}^{*}(s, t) \stackrel{\text { def }}{=} \sup _{y \in \mathbb{R}}\left\{L_{t}^{y}(Y)-L_{s}^{y}(Y)\right\}, \\
L_{X}^{*}(u, v) \stackrel{\text { def }}{=} \sup _{x \in \mathbb{R}}\left\{L_{v}^{x}(X)-L_{u}^{x}(X)\right\}, \\
Y(s, t) \stackrel{\text { def }}{=} \sup _{s \leqslant r \leqslant t} Y(r), \\
\underline{Y}(s, t) \stackrel{\text { def }}{=} \inf _{s \leqslant r \leqslant t} Y(r), \\
Y^{\#}(s, t) \stackrel{\text { def }}{=} \bar{Y}(s, t)-\underline{Y}(s, t) .
\end{gathered}
$$

Clearly, for any $x \in \mathbb{R}$, by (4.10), 


$$
\begin{aligned}
L_{1+\tau_{n}}^{x}(Z)-L_{\tau_{n}}^{x}(Z) & =\int_{\underline{Y}\left(\tau_{n}, 1+\tau_{n}\right)}^{\bar{Y}\left(\tau_{n}, 1+\tau_{n}\right)}\left\{L_{1+\tau_{n}}^{u}(Y)-L_{\tau_{n}}^{u}(Y)\right\} \mathrm{d}_{u} L_{u}^{x}(X) \\
& \leqslant L_{Y}^{*}\left(\tau_{n}, 1+\tau_{n}\right) L_{X}^{*}\left(\underline{Y}\left(\tau_{n}, 1+\tau_{n}\right), \bar{Y}\left(\tau_{n}, 1+\tau_{n}\right)\right) .
\end{aligned}
$$

On the event $E$, we have

$$
\bar{Y}\left(\tau_{0}, 1+\tau_{0}\right) \leqslant \underline{Y}\left(\tau_{1}, 1+\tau_{1}\right) \leqslant \bar{Y}\left(\tau_{1}, 1+\tau_{1}\right) \leqslant \underline{Y}\left(\tau_{2}, 1+\tau_{2}\right) \leqslant \ldots .
$$

Therefore, on $E$ and given $(Y(t) ; t \geqslant 0)$,

$$
\left(\frac{L_{X}^{*}\left(\underline{Y}\left(\tau_{n}, 1+\tau_{n}\right), \bar{Y}\left(\tau_{n}, 1+\tau_{n}\right)\right)}{\left\{Y^{\#}\left(\tau_{n}, 1+\tau_{n}\right)\right\}^{1 / 2}}\right)_{n \geqslant 1}
$$

are i.i.d. variables, distributed as $\sup _{x \in \mathbb{R}} L_{1}^{x}\left(X_{+}\right)$. Accordingly,

$$
\begin{aligned}
\Delta_{7} & \leqslant \mathbb{P}\left(E ; \min _{1 \leqslant n \leqslant N-1} L_{Y}^{*}\left(\tau_{n}, 1+\tau_{n}\right)\left\{Y^{\#}\left(\tau_{n}, 1+\tau_{n}\right)\right\}^{1 / 2} \Xi_{n}>c_{26}(\log T)^{-3 / 4}\right) \\
& \leqslant \mathbb{P}\left(\min _{1 \leqslant n \leqslant m} L_{Y}^{*}\left(\tau_{n}, 1+\tau_{n}\right)\left\{Y^{\#}\left(\tau_{n}, 1+\tau_{n}\right)\right\}^{1 / 2} \Xi_{n}>c_{26}(\log T)^{-3 / 4}\right),
\end{aligned}
$$

where $\left(\Xi_{n}\right)_{n \geqslant 0}$ are i.i.d. variables independent of $\sigma\{Y(t) ; t \geqslant 0\}$, having the same law as $\sup _{x \in \mathbb{R}} L_{1}^{x}\left(X_{+}\right)$. By the strong Markov property,

$$
\Delta_{7} \leqslant \mathbb{P}\left(\min _{1 \leqslant n \leqslant m} \Theta_{n} \Xi_{n}>c_{26}(\log T)^{-3 / 4}\right),
$$

where the $\Theta_{n}$ are distributed as $L_{Y}^{*}(0,1)\left\{Y^{\#}(0,1)\right\}^{1 / 2}$ and we assume that $\left(\Theta_{n}, \Xi_{n} ; n \geqslant 0\right)$ are mutually independent. Write $b \stackrel{\text { def }}{=} b(T)=c_{26}^{2 / 3} /(\log T)^{1 / 2}$. By (4.8) and (4.7),

$$
\begin{aligned}
\mathbb{P}\left\{\Theta_{0} \Xi_{0}<c_{26}(\log T)^{-3 / 4}\right\} & \geqslant \mathbb{P}\left\{\Xi_{0}<b\right\} \mathbb{P}\left(\Theta_{0}<\frac{c_{26}(\log T)^{-3 / 4}}{b}\right) \\
& \geqslant \exp \left(-\frac{c_{25}}{b^{2}}-\frac{c_{25} b^{4}(\log T)^{3}}{c_{26}^{4}}\right) \\
& =\frac{1}{T^{1 / 16}},
\end{aligned}
$$

where we have used (4.9) in the last equality. Going back to (4.12), recalling that $m=\left[T^{1 / 8}\right]$ and (4.9),

$$
\begin{aligned}
\Delta_{7} & \leqslant\left[1-\mathbb{P}\left\{\theta_{0} \Xi_{0}<c_{26}(\log T)^{-3 / 4}\right\}\right]^{m} \\
& \leqslant\left(1-\frac{1}{T^{1 / 16}}\right)^{m} \\
& \leqslant \exp \left(-c_{27} T^{1 / 16}\right) .
\end{aligned}
$$

It remains to estimate $\mathbb{P}\left(E^{c}\right)$. Since $N \leqslant T$, 


$$
\begin{aligned}
\mathbb{P}\left(E^{c}\right) & \leqslant \mathbb{P}(N \leqslant m)+\sum_{n=1}^{[T]} \mathbb{P}\left(\inf _{\tau_{n} \leqslant t \leqslant 1+\tau_{n}} Y(t)<\sup _{\tau_{n-1} \leqslant t \leqslant 1+\tau_{n-1}} Y(t)\right) \\
& =\mathbb{P}\left(\tau_{m}>T-1\right)+[T] \mathbb{P}\left(\inf _{\tau_{1} \leqslant t \leqslant 1+\tau_{1}} Y(t)<\sup _{0 \leqslant t \leqslant 1} Y(t)\right),
\end{aligned}
$$

using the strong Markov property. Since $\tau_{m}=\sum_{n=1}^{m}\left(\tau_{n}-\tau_{n-1}\right)$ and the $\tau_{n}-\tau_{n-1}$ are i.i.d., distributed as $\left(T^{1 / 16}-\mathscr{N}\right)^{2} / \tilde{N}^{2}$ (where $\mathscr{N}$ and $\tilde{N}$ denote as before independent Gaussian $\mathscr{N}(0,1)$ variables $)$, we have

$$
\begin{aligned}
\mathbb{P}\left(\tau_{m}>T-1\right) & \leqslant \mathbb{P}\left(\max _{1 \leqslant n \leqslant m}\left(\tau_{n}-\tau_{n-1}\right)>\frac{T}{2 m}\right) \\
& \leqslant 1-\left\{1-\mathbb{P}\left(\left(T^{1 / 16}-\mathscr{N}\right)^{2}>\frac{T}{2 m} \tilde{N}^{2}\right)\right\}^{m} \\
& \leqslant 1-\left\{1-\mathbb{P}\left(|\mathscr{N}|>\frac{T^{1 / 16}}{2}\right)-\mathbb{P}\left(\frac{9 T^{1 / 8}}{4}>\frac{T}{2 m} \tilde{N}^{2}\right)\right\}^{m} \\
& \leqslant 1-\left\{1-\exp \left(-\frac{T^{1 / 8}}{8}\right)-\frac{3 T^{1 / 16}}{2^{1 / 2}} \frac{m^{1 / 2}}{T^{1 / 2}}\right\}^{m} \\
& \leqslant \frac{c_{28}}{T^{1 / 4}} .
\end{aligned}
$$

On the other hand, by the independence of $\inf _{\tau_{1} \leqslant t \leqslant 1+\tau_{1}} Y(t)$ and $\sup _{0 \leqslant t \leqslant 1} Y(t)$,

$$
\begin{aligned}
\mathbb{P}\left(\inf _{\tau_{1} \leqslant t \leqslant 1+\tau_{1}} Y(t)<\sup _{0 \leqslant t \leqslant 1} Y(t)\right) & =\mathbb{P}\left(T^{1 / 16}-|\mathscr{N}|<|\tilde{N}|\right) \\
& \leqslant \mathbb{P}\left(|\mathscr{N}|>\frac{T^{1 / 16}}{2}\right)+\mathbb{P}\left(|\tilde{N}|>\frac{T^{1 / 16}}{2}\right) \\
& \leqslant 2 \exp \left(-\frac{T^{1 / 8}}{8}\right)
\end{aligned}
$$

Therefore

$$
\mathbb{P}\left(E^{c}\right) \leqslant \frac{c_{28}}{T^{1 / 4}}+2 T \exp \left(-\frac{T^{1 / 8}}{8}\right) \leqslant \frac{c_{29}}{T^{1 / 4}} .
$$

Assembling (4.11), (4.13) and (4.14) yields

$$
\mathbb{P}\left\{\eta\left(\frac{1}{T}\right)>c_{26} T^{-3 / 4}(\log T)^{-3 / 4}\right\} \leqslant \frac{c_{30}}{T^{1 / 4}} .
$$

Take $T_{k} \stackrel{\text { def }}{=} k^{5}$ and apply the Borel-Cantelli lemma to arrive at the following estimate:

$$
\limsup _{k \rightarrow \infty} T_{k}^{3 / 4}\left(\log T_{k}\right)^{3 / 4} \eta\left(\frac{1}{T_{k}}\right) \leqslant c_{26}, \quad \text { a.s. }
$$


Now (4.2) follows by monotonicity of the function $T \mapsto T^{3 / 4}(\log T)^{3 / 4}$.

\section{Acknowledgements}

We are grateful to an anonymous referee for helpful suggestions which led to improvements in the paper.

\section{References}

Bertoin, J. and Shi, Z. (1996) Hirsch's integral test for the iterated Brownian motion. In J. Azéma, M. Emery and M. Yor (eds), Séminaire de Probabilités XXX, pp. 361-368. Lecture Notes Math., 1626. Berlin: Springer-Verlag.

Biane, P. and Yor, M. (1987) Valeurs principales associées aux temps locaux browniens. Bull. Sci. Math., 111, 23-101.

Burdzy, K. (1993) Some path properties of iterated Brownian motion. In E. Çinlar, K.L. Chung and M. Sharpe (eds), Seminar on Stochastic Processes '92, pp. 67-87. Boston, MA: Birkhäuser.

Burdzy, K. and Khoshnevisan, D. (1995) The level sets of iterated Brownian motion. In J. Azéma, M. Emery, P.-A. Meyer and M. Yor (eds), Séminaire de Probabilités XXIX, pp. 231-236. Lecture Notes Math., 1613. Berlin: Springer-Verlag.

Ciesielski, Z. and Taylor, S.J. (1962) First passage times and sojourn times for Brownian motion in space and the exact Hausdorff measure of the sample path. Trans. Amer. Math. Soc., 103, 434-450.

Csáki, E., Csörgö, M., Földes, A. and Révész, P. (1995) Global Strassen type theorems for iterated Brownian motion. Stochastic Processes Applic., 59, 321-341.

Csáki, E., Csörgö, M., Földes, A. and Révész, P. (1996) The local time of iterated Brownian motion. $J$ Theor. Probab., 9, 717-743.

Csáki, E., Csörgő, M., Földes, A. and Révész, P. (1997a) On the occupation time of an iterated process having no local time. Stochastic Processes Applic., 70, 199-217.

Csáki, E. and Földes, A. (1986) How small are the increments of the local time of a Wiener process? Ann. Probab., 14, 533-546.

Csáki, E. and Földes, A. (1997) On the logarithmic average of iterated processes. Statist. Probab. Lett., 33, 347-358.

Csáki, E., Földes, A. and Révész, P. (1997b) Strassen theorems for a class of iterated processes. Trans. Amer. Math. Soc., 349, 1153-1167.

Csörgö, M. and Révész, P. (1979) How small are the increments of a Wiener process? Stochastic Processes Applic., 8, 119-129.

Eisenbaum, N. (1990) Un théorème de Ray-Knight lié au supremum des temps locaux browniens. Probab. Theory Related Fields, 87, 27-34.

Getoor, R.K. (1979) The Brownian escape process. Ann. Probab., 5, 864-867.

Hu, Y. (1996) Sur le mouvement brownien: calcul de lois; études asymptotiques; filtrations; relations avec certaines équations paraboliques. Thèse de Doctorat, Université Paris VI.

Kesten, H. (1965) An iterated logarithm law for local time. Duke Math. J., 32, 447-456.

Khoshnevisan, D. and Lewis, T.M. (1997) Stochastic calculus for Brownian motion on a Brownian fracture. Ann. Appl. Probab. To appear. 
Khoshnevisan, D., Lewis, T.M. and Li, W.V. (1994) On the future infima of some transient processes. Probab. Theory Related Fields, 99, 337-360.

Lévy, P. (1939) Théorie de l'Addition des Variables Aléatoires. Paris: Gauthier-Villars.

Révész, P. (1990) Random Walk in Random and Non-Random Environments. Singapore: World Scientific.

Revuz, D. and Yor, M. (1994) Continuous Martingales and Brownian Motion, 2nd edn. Berlin: Springer-Verlag.

Rogers, L.C.G. and Williams, D. (1987) Diffusions, Markov Processes, and Martingales, Vol. II, Itô Calculus. Chichester, West Sussex: Wiley.

Shi, Z. and Yor, M. (1997) Integrability and lower limits of the local time of iterated Brownian motion. Studia Sci. Math. Hung., 33, 279-298.

Xiao, Y. (1997) Local times and related properties of multi-dimensional iterated Brownian motion. J. Theor. Probab., 11, 383-408.

Yor, M. (1992) Some Aspects of Brownian Motion, Part I, Some Special Functionals. Lectures in Math. Eidgenössiche Technische Hochschule Zürich. Basel: Birkhäuser.

Received February 1997 and revised September 1997 